\title{
High incidence of hypertriglyceridemia in a Brazilian cohort of people living with HIV/AIDS undergoing antiretroviral treatment in Belo Horizonte, 2001-2010
}

\author{
Cássia Cristina Pinto Mendicino ${ }^{[1]}$, Letícia Pena Braga ${ }^{[2]}$, Cristiane Aparecida Menezes de \\ Pádua $^{[2]}$ and Mark Drew Crosland Guimarães ${ }^{[1]}$
}

\begin{abstract}
[1].Programa de Pós-Graduação em Saúde Pública, Faculdade de Medicina, Universidade Federal de Minas Gerais, Belo Horizonte, Minas Gerais, Brasil.[2]. Programa de Pós-Graduação em Medicamentos e Assistência Farmacêutica, Faculdade de Farmácia, Universidade Federal de Minas Gerais, Belo Horizonte, Minas Gerais, Brasil.
\end{abstract}

\begin{abstract}
Introduction: Hypertriglyceridemia incidence should be estimated in HIV-infected patients after antiretroviral therapy (ART) initiation. Methods: We retrospectively analyzed clinical data of HIV-infected adults at 3 public referral centers. Cumulative and person-time incidences were estimated for patients without hypertriglyceridemia. Survival time and hazard ratio (HR) were estimated by Kaplan-Meier analysis and Cox proportional regression, respectively. Results: Cumulative and person-time incidences were $40.4 \%$ and 1.4 cases/100 person-months, respectively. The median period for hypertriglyceridemia occurrence was 47 months. Men and patients with switched ART regimens had increased hypertriglyceridemia risk (HR=3.05 and 3.34, respectively). Conclusions: Hypertriglyceridemia incidence is high in HIV-infected patients undergoing ART.
\end{abstract}

Keywords: HIV/AIDS. Hypertriglyceridemia. ART.

The introduction of antiretroviral therapy (ART) for control of human immunodeficiency virus/acquired immune deficiency syndrome (HIV/AIDS) has allowed viral suppression and immune recovery, leading to long-term survival and better quality of life among the affected population ${ }^{(1)}$. HIV/AIDS has thus become a manageable chronic illness, and current guidelines worldwide recommend early ART initiation ${ }^{(2)}$. However, long-term exposure to ART potentially increases the risk of undesirable adverse effects, including metabolic changes ${ }^{(3)}$.

Among the various metabolic disorders prevalent in HIV/ AIDS patients undergoing ART, dyslipidemias stand out for their high incidence, atherogenic capacity, and potential to cause cardiovascular diseases ${ }^{(3)}$. In addition, in persons living with HIV/AIDS (PLHA), dyslipidemias can occur before and/ or after initiation of ART ${ }^{(4)}$. In PLHA who have not yet started ART, dyslipidemia is mainly characterized by early decrease in the high-density lipoprotein (HDL) cholesterol levels and an increase in the triglyceride levels in more advanced stages of infection ${ }^{(4)}$. After ART initiation, PLHA develop dyslipidemia mainly due to the exposure to the ART drugs, and at this stage, dyslipidemia is

Corresponding author: Dra. Cássia Cristina Pinto Mendicino.

e-mail: cassiamendicino@gmail.com

Received 10 March 2016

Accepted 30 August 2016 mostly characterized by an increase in both triglycerides and HDL cholesterol levels ${ }^{(5)}$. Hypertriglyceridemia is the most common lipid alteration that occurs after ART initiation and is an important dyslipidemia marker in PLHA undergoing $\mathrm{ART}^{(6)}$. However, only a few thus far studies have assessed the magnitude of this problem in public AIDS referral centers in Brazil. Therefore, this study aimed at estimating the incidence of hypertriglyceridemia and its potential contributing factors in a cohort of PLHA after ART initiation.

This study was a prospective non-concurrent study of adult (age $\geq 18$ years) HIV/AIDS patients who began ART between 2001 and 2005. Medical charts and laboratory data of patients attending the 3 main HIV/AIDS public referral centers in Belo Horizonte-MG, Brazil, were reviewed up to 5 years after the first ART prescription. Data were collected from November 2012 through September 2013. The study was approved by the Institutional Review Boards of the Federal University of Minas Gerais and by the participating centers.

Patients with available results of serum triglyceride levels at baseline ( \pm 3 months between the date of the exam and the date of first ART) and during follow-up were included, whereas prevalent cases of hypertriglyceridemia were excluded. For this analysis, the outcome of interest was the presence of hypertriglyceridemia, identified as the result of triglyceride level $\geq 150 \mathrm{mg} / \mathrm{dL}$ during follow-up. Follow-up duration was defined as the period between the date of the first ART prescription 
and the date of the first occurrence of hypertriglyceridemia or the last medical visit for persons without hypertriglyceridemia. Data on the potential explanatory variables (socio-demographic, behavioral, health services, and clinics) were collected at baseline.

The absolute and relative frequencies were estimated to characterize the study population. Cumulative and person-time incidences of hypertriglyceridemia were estimated. Survival time was also estimated by the Kaplan-Meier method, with $95 \%$ confidence intervals (CIs). Multivariate analysis was performed using Cox proportional hazard model. The magnitude of the associations was estimated by the hazard ratio (HR), with $95 \%$ CIs. The assumption of risk proportionality was assessed by Schoenfeld residual analysis. Statistical analysis was performed using R software version 3.0.1.
Among 247 patients initially selected, 100 (40.5\%) had available serum triglycerides results. Of these, $40 \%$ were prevalent cases of hypertriglyceridemia $(n=40)$.

Three patients who did not have at least 3 months of follow-up were excluded; thus, finally, 57 patients without hypertriglyceridemia were available for follow-up. Table 1 shows the descriptive analysis of the study population: $56.8 \%$ of patients started ART CD4 T lymphocytes $\leq 200$ cells $/ \mathrm{mm}^{3}$ and $59.6 \%$ showed AIDS-defining illness or clinical signs of AIDS.

We noted 23 (40.4\%) new cases of hypertriglyceridemia, totaling to 1.4 cases of hypertriglyceridemia per 100 personmonths. The median follow-up duration was 27 months. Results of the univariate and multivariate analyses are shown in Table $\mathbf{1}$.

TABLE 1

Univariate and multivariate analyses of hypertriglyceridemia according to selected variables in Belo Horizonte, Minas Gerais, Brazil.

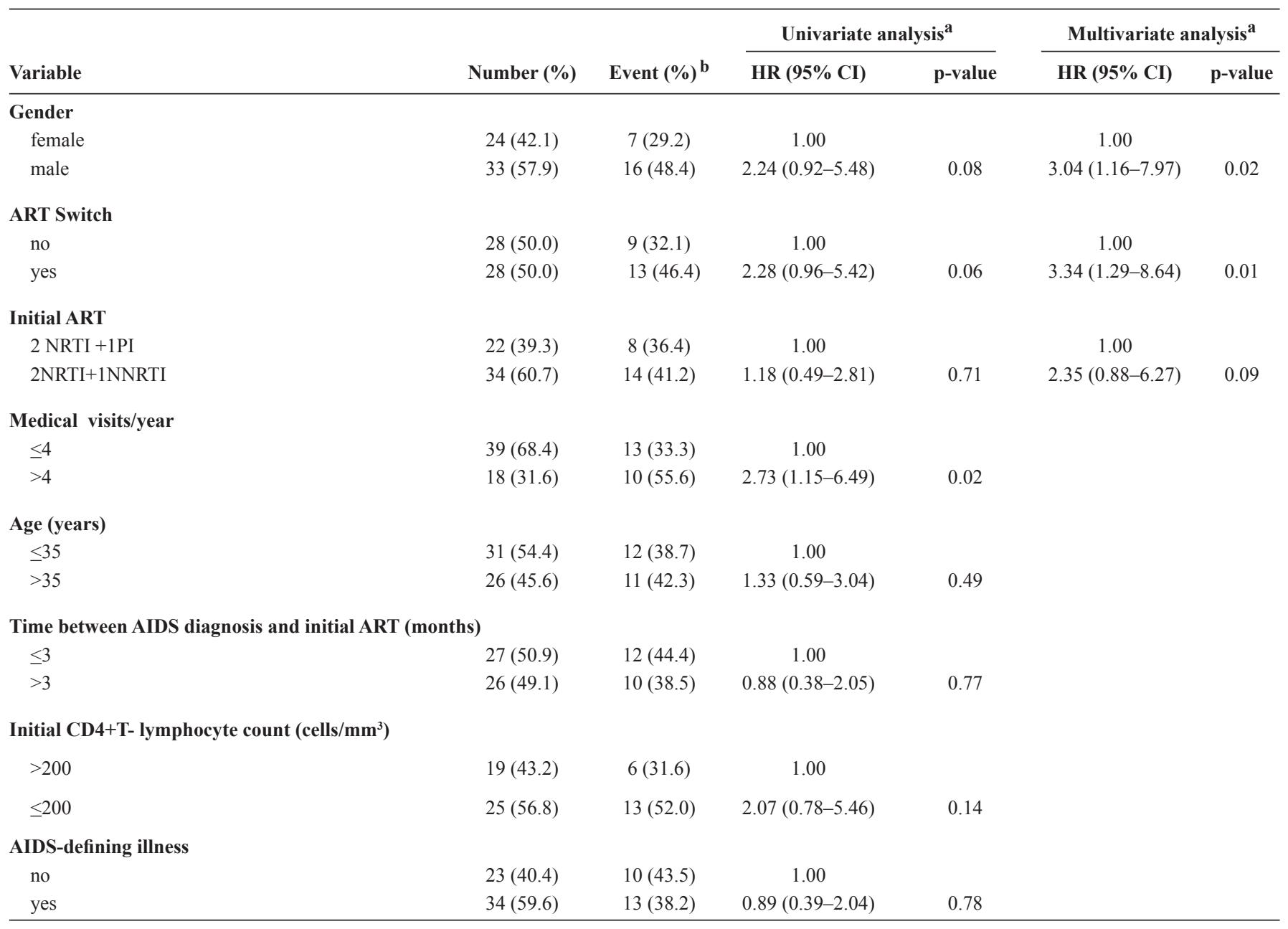

HR: hazard ratio; CI: confidence interval; ART: antiretroviral therapy; NRTI: nucleoside reverse transcriptase inhibitor; PI: protease inhibitor; NNRTI: non-nucleoside reverse transcriptase inhibitor; AIDS: acquired immune deficiency syndrome.

Missing values were excluded.

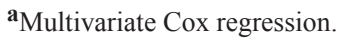

$\mathbf{b}^{\mathbf{b}}$ Event (\%): Incident cases of hypertriglyceridemia by category.

The Schoenfeld residual analysis showed presence of proportionality of risk (p-values: (gender, 0.37; ART switch, 0.70; initial ART, 0.88; global,0.74). 


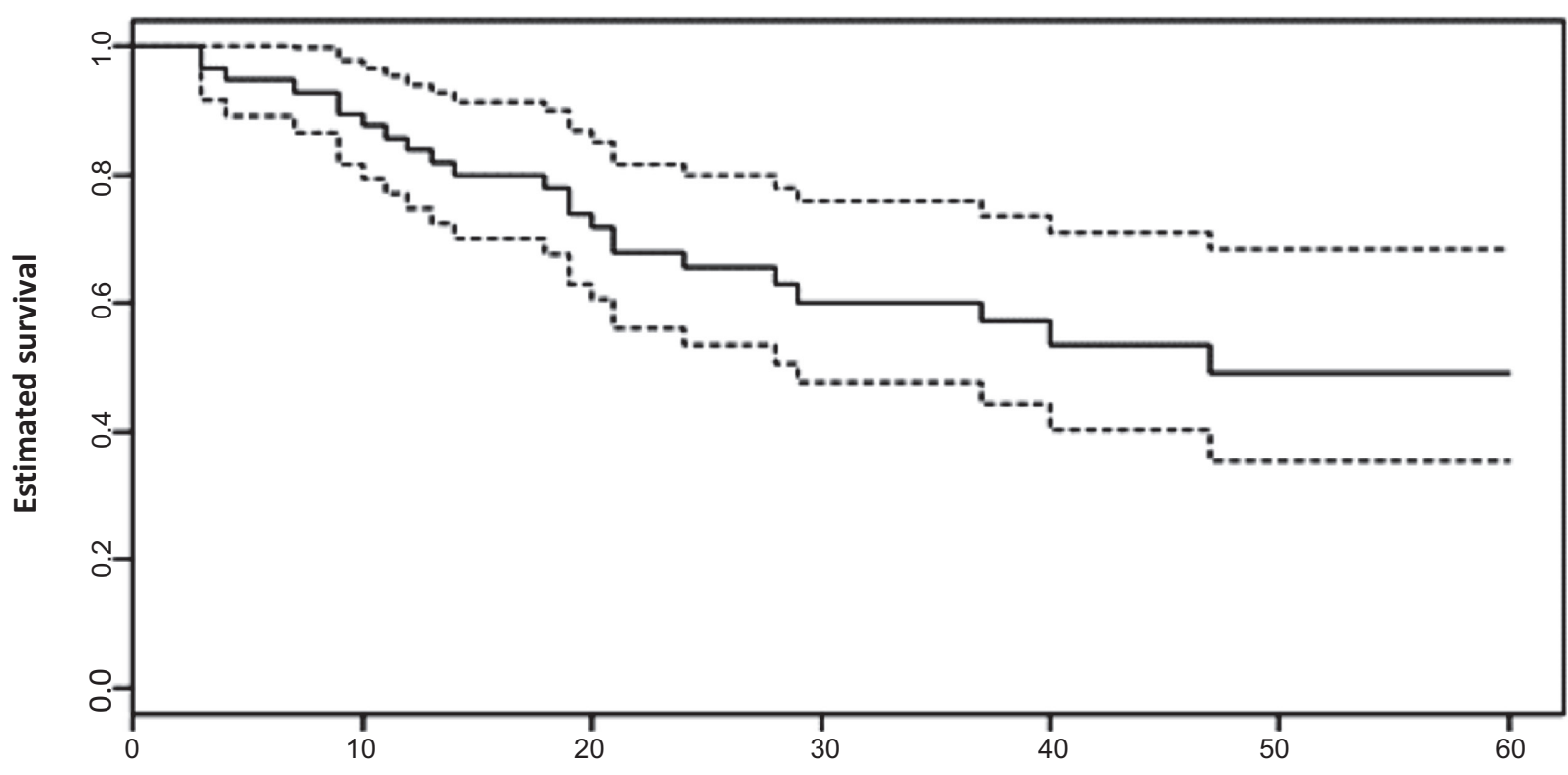

Time (months)

FIGURE 1. Kaplan-Meier curve of median time for the occurrence of hypertriglyceridemia, with $95 \%$ confidence intervals, for 57 HIV/AIDS participants who started ART between 2001 and 2005 in 3 treatment centers in Belo Horizonte, Minas Gerais, Brazil. HIV/AIDS: human immunodeficiency virus/acquired immune deficiency syndrome; ART: antiretroviral therapy.

Gender and ART switching during follow-up remained statistically significant $(\mathrm{p}<0.05)$ in the final multivariate analysis, whereas initial ART regimen was retained in the final model due to its clinical significance. Men and patients with switched ART regimens during follow-up were more likely to develop hypertriglyceridemia ( $\mathrm{HR}=3.04$ and 3.44 , respectively). The final model explained $70.0 \%$ of the concordance and $16.7 \%$ of the variability, and the Schoenfeld residuals analysis indicated proportionality of risks for each variable (Table 1). Figure 1 shows the overall Kaplan-Meier survival curve. At 12 and 24 months, approximately 84\% (95\% CI: 74-94\%) and 65\% (95\% CI: $54-80 \%$ ) of the patients did not have hypertriglyceridemia. The median time until the occurrence of hypertriglyceridemia (incident cases) was estimated to be 47 months.

The estimated prevalence of hypertriglyceridemia at baseline $(40 \%)$ in this study was higher than the estimates among the general adult population in the United States from 1999 to 2004 $(33 \%)^{(7)}$ and the prevalence of hypertriglyceridemia in PLHA (naïve ART patients) (31\%) in Ethiopia from September 2011 to May $2012^{(8)}$. Possible explanations include the predominance of male patients and the high proportion of patients with late initiation of ART in our study.

We also found a high incidence of hypertriglyceridemia (cumulative incidence of $40.4 \%$ in only 27 months of follow-up) in our cohort of patients undergoing ART in HIV/AIDS public referral centers in Brazil. A retrospective study conducted from January 2008 to May 2011 with 498 patients in Brazil found a cumulative incidence of hypertriglyceridemia of $29.8 \%$ (follow-up time, 36 months) ${ }^{(9)}$. Furthermore, Calza et al ${ }^{(10)}$ followed up 220 patients, who began ART between January 1998 and December 2000 in Italy, for 12 months and estimated a cumulative incidence of $38 \%{ }^{(10)}$. However, the great variation in study design and eligibility criteria of different studies should be taken into account.

During follow-up, the levels of triglycerides showed a discreet but statistically significant increase, from $101 \mathrm{mg} / \mathrm{dL}$, in the beginning of the study to $134 \mathrm{mg} / \mathrm{dL}$ at the end of followup $(p<0.01)$. These results are similar to those reported by Quercia et $\mathrm{al}^{(11)}$ in a multicenter clinical trial, where in PLHA began treatment between 2009 and 2011. In their study, the average triglyceride level was $114.7 \mathrm{mg} / \mathrm{dL}$ before starting ART $(\mathrm{n}=954)$ and increased to $123.4 \mathrm{mg} / \mathrm{dL}$ after 12 months of ART $(\mathrm{n}=836)^{(11)}$.

Hypertriglyceridemia is a result of the combination of genetic and behavioral factors, and the triglyceride levels are, in general, higher in men than in women ${ }^{(7)}$. However, some studies have associated hypertriglyceridemia in PLHA with the male gender ${ }^{(12)}$, while other studies have shown no such relationship ${ }^{(9)}$. Our findings corroborate those of a previous study on 372 PLHA undergoing ART, which showed a 2.2 times increased risk of hypertriglyceridemia in men $^{(12)}$.

Alterations in the lipid profile are associated with the use of the major available classes of antiretrovirals (ARs). The most frequent hypertriglyceridemia occurs with protease inhibitor (PI) regimens, which contain ritonavir ${ }^{(13)}$. In our study, patients who began ART with a combination of two analog reverse 
transcriptase inhibitors nucleoside and a reverse transcriptase inhibitor non-nucleoside analogue had an increased risk of hypertriglyceridemia as compared to those who began treatment with PI-containing regimens, although this difference was not statistically significant in the final model $(\mathrm{p}=0.09)$. One potential explanation is that our study only considered the initial ART and during follow-up, only $5.3 \%$ of the population used ART regimens including ritonavir at low dosages along with other PI such as indinavir or atazanavir. The infrequent use of ritonavir and ART switching during follow-up may partially explain the lack of association between the type of ART and the occurrence of hypertriglyceridemia in this study.

In our study, ART switching was associated with the occurrence of hypertriglyceridemia. Adverse effects mainly including anemia, hypersensitivity, and gastrointestinal intolerance are the most frequent causes of ART switching ${ }^{(13)}$. In addition, adverse reactions are very common when treating opportunistic diseases (a marker of more advanced stages of the infection) alongside ART ${ }^{(13)}$. It is also possible that ART switching may be a consequence rather than a cause of hypertriglyceridemia. As such, further studies are needed to clarify this issue.

Despite our important findings, our study has some limitations. First, it is not common among health professionals to request triglyceride tests, and these tests were mandated only in more advanced stages of infection, with important variation between the health facilities. Second, the study sample initially comprised 247 patients, but the number reduced to 100 patients, as we only selected patients with triglyceride levels available at baseline. However, the population with available triglyceride levels had a higher number of medical visits per year than those without available triglyceride levels $(p<0.05)$ (data not shown). Further, a high number of medical visits are usually related to advanced stage of infection ${ }^{(14)}$, which can contribute to the increased triglyceride levels, thus potentially overestimating the occurrence of hypertriglyceridemia. Finally, the small sample size available for analysis may have caused a lack of statistical power for the selected explanatory variables.

Due to the long duration of ART, hypertriglyceridemia may persist as an important adverse effect in patients undergoing ART, which may burden for both health services and patients. Usually, more than one drug is necessary to treat dyslipidemia in PLHA undergoing ART, especially when they have hypertriglyceridemia, and this could increase the costs and cause adherence problems for the affected people ${ }^{(4)}$. In addition, studies have shown that the available triglyceridereducing drugs do not have the same effectiveness in PLHA undergoing ART as they do in PLHA not undergoing ART due to drug-interactions with $\mathrm{ARs}^{(4)}$. Thus, new classes of ARs such as integrase inhibitors (e.g., raltegravir) and chemokine receptor antagonist (CCR5 inhibitors) (e.g., maraviroc) are potential options to reduce the risk of dyslipidemia in this population ${ }^{(15)}$. Furthermore, monitoring PLHA undergoing ART for long-term adverse effects should be included in medical care at these public referral centers.

\section{Acknowledgments}

The authors are grateful to the Grupo de Pesquisa e Avaliação em Saúde (GPEAS/UFMG), Centro de Treinamento e Referência em Doenças Infecto parasitárias - CTR-DIP Orestes Diniz, Secretaria Municipal de Saúde de Belo Horizonte (Brasil) - SMSA/BH e UFMG; Hospital Eduardo de Menezes - HEM, Fundação Hospitalar do Estado de Minas Gerais - FHEMIG e Centro de Aconselhamento e Testagem Sagrada Família, SMSA/BH.

\section{Conflict of Interest}

The authors declare that they have no conflict of interest.

\section{Financial Support}

Fundação de Amparo à Pesquisa do Estado de Minas Gerais (FAPEMIG): Project number CDS-APQ-00043-10. Conselho Nacional de Desenvolvimento Científico e Tecnológico (CNPq): Project number 484865/2011-0.

\section{REFERENCES}

1. Nosyk B, Montagner JSG, Colley G, Lima VD, Chan K, Heath $\mathrm{K}$, et al. The cascade of HIV care in British Columbia, Canada, 1996-2011: a population-based retrospective cohort study. Lancet Infect Dis 2014; 14:40-49.

2. Ministério da Saúde. Secretaria de Vigilância em Saúde. Departamento de DST, Aids e Hepatites Virais. Protocolo clínico de diretrizes terapêuticas para o manejo da infecção pelo HIV em adultos. Brasília: Ministério da Saúde; 2013. 227p.

3. Grinspoon S, Carr A. Cardiovascular risk and body-fat abnormalities in HIV-infected adults. N Eng J Med 2005; 352:48-62.

4. Grunfeld C. Dyslipidemia and its treatment in HIV infection. Top HIV Med 2010; 18:112-118.

5. Denue BA, Alkali MB, Abjah AU, Kida IM, Ajayi B, Fate BZ. Changes in lipid profiles and other biochemical parameters in HIV-1 infected patients newly commenced on HAART regimen. Infec Dis Res Treat 2013;6:7-14.

6. Lu CL, Lin YH, Wong WW, Lin HH, Ho MW, Wang NC, etal. Outcomes of switch to atazanavir-containing combination antiretroviral therapy in HIV-1-infected patients with hyperlipidemia. J Microbiol Immunol and Infect 2011; 44:258-264.

7. Ford ES, Li C, Zhao G, Pearson WS, Mokdad AH. Hypertriglyceridemia and its pharmacologic treatment among US adults. Arch Intern Med 2009; 169:572-578.

8. Abebe M, Kinde S, Belay G, Gebreegziabxier A, Challa F, Gebeyehu T. et al. Antiretroviral treatment associated hyperglycemia and dyslipidemia among HIV infected patients at Burayu Health Center, Addis Ababa, Ethiopia: a cross-sectional comparative study. BMC Res Notes 2014; 7:380.

9. Pinto Neto LF, Neves MB, Ribeiro-Rodrigues R, Page K, Miranda AE. Dyslipidemia and fasting glucose impairment among HIV patients three years after the first antiretroviral regimen in a Brazilian AIDS outpatient clinic. Braz J Infect Dis 2013;17:438-443.

10. Calza L, Manfredi R, Farneti B, Chiodo F. Incidence of hyperlipidemia in a cohort of $212 \mathrm{HIV}$-infected patients receiving a protease inhibitor-based antiretroviral therapy. Int J Antimicrob Agents 2003; 22:54-59.

11. Quercia R, Roberts J, Martin-Carpenter L, Zala C. Comparative changes of lipid levels in treatment-naive, HIV-1-infected adults treated with dolutegravir vs. efavirenz, raltegravir, and ritonavir- 
boosted darunavir-based regimens over 48 weeks. Clin Drug Investig 2015;35:211-219.

12. de Araújo PSR, de Alencar Ximenes RA, Lopes CFC, Duarte JY, da Silva MM, Carneiro EM. Antiretroviral treatment for HIV infection/AIDS and the risk of developing hyperglycemia and hyperlipidemia. Rev Inst Med Trop Sao Paulo 2007; 49:73-78.

13. Cesar C, Shepherd BE, Krolewiecki AJ, Fink VI, Schechter M, Tuboi SH, et al. Rates and reasons for early change of first HAART in HIV-1-infected patients in 7sites throughout the Caribbean and Latin America. PLoS One 2010; 5:e10490.

14. Acúrcio FA, Cesar CC, Guimarães MD. Health care utilization and survival among patients with AIDS in Belo Horizonte, Minas Gerais, Brazil. Cad Saude Publica1998; 14:811-820.

15. Feeney ER, Mallon PWG. HIV and HAART-associeted dyslipidemia. Open Cardiovasc Med J 2001; 5:49-63. 